\title{
Predictive Testing for Multiple Endocrine Neoplasia Type 1 Using DNA Polymorphisms
}

\author{
Catharina Larsson," Joseph Shepherd,‡ Yusuke Nakamura,` Carl Blomberg, ${ }^{*}$ Günther Weber," Barbro Werelius, ${ }^{*}$ Nick

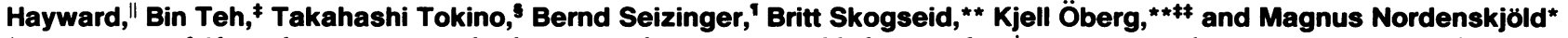 \\ ${ }^{*}$ Department of Clinical Genetics, Karolinska Hospital, S-10401 Stockholm, Sweden; ${ }^{\ddagger}$ Department of Surgery, University of Tasmania, \\ Hobart, Australia; ${ }^{\S}$ Division of Biochemistry, Cancer Institute, Tokyo, Japan; "Queensland Institute of Medical Research, Brisbane, \\ Qld, Australia; 'Molecular Neuro-Oncology Laboratory, Massachusetts General Hospital, Boston, Massachusetts; ${ }^{* *}$ Department of \\ Internal Medicine, University Hospital, Uppsala, Sweden; and ${ }^{\ddagger}$ Ludwig Institute for Cancer Research, \\ University Hospital, Uppsala, Sweden
}

\begin{abstract}
Multiple endocrine neoplasia type 1 (MEN1) is an autosomal dominantly inherited predisposition to neoplastic lesions of the parathyroids, pancreas, and the pituitary. We have previously located the predisposing genetic defect to the long arm of chromosome 11 by genetic linkage. In this study, 124 members of six MEN1 families, including 59 affected individuals, were genotyped for restriction fragment length polymorphisms with different DNA probes, and the genetic linkage between these marker systems and MEN1 was determined. 13 marker systems (17 DNA probes) were found to be linked to MEN1. These markers are located within a region on chromosome 11 spanning 14\% meiotic recombinations, with the MEN1 locus in the middle. Four of the marker systems are on the centromeric side of MEN1, and four on the telomeric side, based on meiotic crossovers. The remaining five DNA probes are closely linked to MEN1, with no crossovers in our set of families. The 13 marker systems can be used for an accurate and reliable premorbid test for MEN1. In most clinical situations it is possible to identify a haplotype of this part of chromosome 11 with the mutant MEN1 allele in the middle. The calculated predictive accuracy is $>\mathbf{9 9 . 5 \%}$ if three such marker systems are informative. Therefore, genetic linkage testing can be used for informed genetic counseling in MEN1 families, and to avoid unnecessary biochemical screening programs. (J. Clin. Invest. 1992. 89:1344-1349.) Key words: cancer predisposition • chromosome 11 - genetic linkage $\bullet$ restriction fragment length polymorphism • tumor suppressor gene
\end{abstract}

\section{Introduction}

Multiple endocrine neoplasia type $1(\mathrm{MEN} 1)^{1}$ is a genetic disorder characterized by neoplastic lesions in the parathyroid glands, the neuro endocrine pancreas, and the anterior lobe of

Address correspondence and reprint requests to Dr. Catharina Larsson, Dept. of Clinical Genetics, Karolinska Hospital, S-104 01 Stockholm, Sweden.

Received for publication 01 July 1991 and in revised form 30 September 1991 .

1. Abbreviations used in this paper: MEN 1, multiple endocrine neoplasia type 1; RFLP, restriction fragment length polymorphism.

J. Clin. Invest.

(c) The American Society for Clinical Investigation, Inc.

0021-9738/92/04/1344/06 \$2.00

Volume 89, April 1992, 1344-1349 the pituitary gland $(1,2)$. We previously mapped the MEN1 locus to chromosomal region $11 \mathrm{q} 11-\mathrm{q} 13$ by genetic linkage to restriction fragment length polymorphism (RFLP) markers in families segregating the disease (3). By identifying meiotic crossovers in MEN1 families, we were able to locate the disease gene close to the PYGM locus and flanked by the D11S288 locus located $7 \mathrm{cM}$ on the centromeric side, and by INT $27 \mathrm{cM}$ on the telomeric side of this marker (4)(Fig. 1). Furthermore, comparisons of the genotypes in normal and tumor tissue from the same individual showed that the pathogenesis of pancreatic, as well as parathyroid lesions, involve unmasking of a recessive mutation at the MEN1 locus $(3,5-8)$, in agreement with the two-mutational model originally postulated by Knudson (9). Deletion mapping of two MEN1-associated lesions suggested that the MEN1 locus is telomeric to PYGM, and within the 5 cM interval between PYGM and D11S146 $(4,7)$.

Based on the autosomal dominant mode of inheritance, offspring of MEN1 patients have a risk of close to $50 \%$ of developing the disease. Affected individuals may present with any of the MEN1-associated lesions in early teenage, or escape clinical symptoms for several decades. Children of MEN1 patients usually undergo repeated biochemical screenings in order to identify lesions as early as possible (10). However, since MEN1-associated lesions may develop slowly, unaffected relatives at risk cannot be excluded as late onset gene carriers until the age of $35 \mathrm{yr}$, despite extensive biochemical testing. Hence, there is a call for informed genetic counseling in MEN1 families, and an avoidance of unnecessary biochemical screening tests. We have attempted to develop a reliable and clinically useful predictive testing for MEN1 based on genetic linkage to DNA markers.

Such testing procedures can make use of the detailed genetic mapping data recently generated for the chromosomal region 11q12-q13. This involves isolation of new genetic markers which are then ordered by genetic linkage in reference families, physically, on somatic cell hybrids, or by long-range restriction mapping using pulsed-field gel electrophoresis (4, 11-14). We have tested genetic linkage to the disease using a number of DNA probes which we have mapped physically to the chromosome region harboring the MEN1 gene. Extensive family material with 59 MEN1 cases from five Swedish families and one large kindred from Tasmania was analyzed. In total, 13 marker systems (17 probes) showed significant linkage to MEN1. Of these, 11 probes had not previously been tested for linkage to the disease.

Five of these probes are located close to the disease gene with no meiotic crossovers detected, while four marker systems flank MEN 1 on the centromeric side, and four marker systems 
are telomeric. The accuracy and usefulness of DNA-based testing for MEN1 predisposition is illustrated in a set of families suitable for such diagnosis.

\section{Methods}

Clinical cases. Three of the five Swedish families with MEN1 (KA, EZ, $\mathrm{LU}$ ) have been described elsewhere (3). The AX and RA families were referred for clinical testing, and are shown in Fig. 2. In addition to these five families, part of one large MEN1 pedigree first reported by Shep- herd in 1985 from Tasmania was studied (15). This family has previously not been studied by linkage. By the end of 1989 , over 150 affected individuals from 2,300 family members spanning eight generations had been traced back to a woman who came to Tasmania in the middle of the last century (16). Her description recorded by the ship's surgeon is consistent with the diagnosis of MEN1. The scoring for MEN1 was conservative in order to avoid incorrect diagnosis. Unaffected individuals at risk were scored as "normal" only if they had passed the age of 35 years and had undergone repeated and extensive biochemical screenings (10) with no signs of the disease, while offsprings to MEN1 cases who did not fulfill these criteria were scored as "unknown."

Table I. DNA Markers Linked to the MEN1 Locus

\begin{tabular}{|c|c|c|c|c|c|c|}
\hline $\begin{array}{l}\text { Locus } \\
\text { name }\end{array}$ & $\begin{array}{l}\text { Probe } \\
\text { name }\end{array}$ & Enzyme & $\begin{array}{l}\text { Constant } \\
\text { bands }\end{array}$ & $\begin{array}{l}\text { Allele } \\
\text { sizes }\end{array}$ & $\begin{array}{l}\text { Allele } \\
\text { freq. }\end{array}$ & $\begin{array}{c}\text { Observed } \\
\text { heterozygosity }\end{array}$ \\
\hline \multirow[t]{2}{*}{ D11S288 } & $\mathrm{p} 3 \mathrm{C} 7$ & MspI & & 5.7 & 0.72 & \\
\hline & & & & 3.1 & 0.28 & \\
\hline \multirow[t]{2}{*}{ D11S149 } & pTHH26 & PvulI & & 5.2 & 0.14 & \\
\hline & & & & 3.2 & 0.84 & \\
\hline \multicolumn{4}{|c|}{ Haplotype for markers D11S288 and D11S149 } & & & $65 \%$ *‡ \\
\hline \multirow[t]{2}{*}{$\mathrm{CD} 20$} & $\mathrm{pB} 1-21$ & MspI & 2.8 & 9.0 & 0.47 & \\
\hline & & & & 6.0 & 0.53 & \\
\hline \multirow[t]{2}{*}{ D11S480 } & cCI11-319 & PstI & several & 3.0 & 0.50 & \\
\hline & & & & 1.4 & 0.50 & \\
\hline \multirow[t]{5}{*}{ PGA } & pcAGP9 & EcoR1 & & 19.0 & 0.06 & \\
\hline & & & & $16.6+15+3.5$ & 0.37 & \\
\hline & & & & $16.6+15+12+3.5$ & 0.54 & \\
\hline & cCLPGA $12^{\S}$ & PvulI & $4.4 ; 3.4 ; 3.0 ; 2.8$ & 11 & 0.37 & \\
\hline & & & $2.1 ; 1.9 ; 1.4 ; 1.3 ; 1.1$ & $6+5$ & 0.63 & \\
\hline \multirow[t]{4}{*}{ PYGM } & $\mathrm{pMCMP}^{\S}$ & Mspl & & $2.2-2.5$ & & $58 \%$ \\
\hline & & & & 5 alleles VNTR & & \\
\hline & $\mathrm{cCL} 5^{\S}$ & EcoR1 & $14 ; 6.6 ; 5.4$ & 19.0 & 0.11 & \\
\hline & & & & 17.5 & 0.89 & \\
\hline \multirow[t]{2}{*}{ D11S427 } & cCI1 $1-4^{\S}$ & EcoR1 & $21 ; 16$ & $9.0-9.6$ & & $60 \%$ \\
\hline & & & & 4 alleles VNTR & & \\
\hline \multirow[t]{2}{*}{ D11S750 } & cCLGW $4^{\S}$ & SacI & $11.3 ; 6.4 ; 4.4$ & 3.6 & 0.80 & \\
\hline & & & $1.6,1.5 ; 1.3$ & 2.4 & 0.20 & \\
\hline \multirow[t]{2}{*}{ D11S449 } & cCI11-219 & PvulI & several & 3.7 & 0.33 & \\
\hline & & & & 3.0 & 0.67 & \\
\hline \multirow[t]{2}{*}{ D11S97 } & pMS51 & HaeIII & & $1.3-4.3$ & & $89 \%$ \\
\hline & & & & 9 alleles VNTR & & \\
\hline \multirow[t]{4}{*}{ D11S146 } & pHBI59 & TaqI & & 1.2 & 0.55 & \\
\hline & & & & 0.8 & 0.45 & \\
\hline & cCL59 & EcoRV & $24 ; 18 ; 7.4$ & 7.0 & 0.22 & \\
\hline & & & & 6.4 & 0.78 & \\
\hline \multicolumn{3}{|c|}{ Haplotype for marker D11S146 } & & & & $55 \% * \ddagger$ \\
\hline \multirow[t]{4}{*}{ D1 1S751 } & cCL- $8^{\S}$ & EcoRV & $20 ; 5.2 ; 2.2$ & 6.6 & 0.50 & \\
\hline & & & & 5.8 & 0.50 & \\
\hline & clone 8 & EcoR1 & & 7.4 & 0.89 & \\
\hline & & & & 6.7 & 0.11 & \\
\hline \multicolumn{3}{|c|}{ Haplotype for marker D11S751 } & & & & $60 \% * \ddagger$ \\
\hline \multirow[t]{4}{*}{ INT2 } & SS6 & TaqI & & 4.1 & 0.60 & \\
\hline & & & & 2.3 & 0.40 & \\
\hline & & BamHI & & 8.4 & 0.63 & \\
\hline & & & & $5.6+2.8$ & 0.37 & \\
\hline \multicolumn{4}{|c|}{ Haplotype for marker INT2 } & & & $60 \%^{\ddagger}$ \\
\hline
\end{tabular}

* Combined haplotype of two probes. ${ }^{\ddagger}$ Observed in the present study. Loci in bold indicate anchor markers previously mapped on reference families. ${ }^{\S}$ Indicates probes with repetitive elements for which human DNA was included in the hybridization. 
DNA probes, genotyping, and linkage analysis. The probes used and the RFLP's detected with these markers are described in Table I. D11S288 (17), D11S149 (17), PYGM/pMCMP1 (17), and D11S146/ pHBI59 (17) are available from American Type Culture Collection (Rockville, MD). CD20 (18) was obtained from T.F. Tedder, PGA/ pcAGP9 from B. Zelle, D11S97 (18) from ICI, INT2 (18) from N. Spurr, and clone 8/D11S751 $(14,19)$ from T. Rabbits. The newly isolated cosmid clones for D11S480, D11S427, and D11S449 have been published (12). Table I includes only those 17 probes which showed significant linkage to the MEN1 locus (see below). For 10 of the probes listed previously published RFLPs existed $(12,17,18)$. New allele systems were identified through locus expansion in cosmids for PGA (cCLPGA12), PYGM (cCL15), D11S146 (cCL59), and D11S751 (cCL8), and the new cosmid D11S750/cCLGW4 was isolated from a library constructed from radiation-reduced somatic cell hybrids containing the 11q11-q13 region (14).

The gene order given for the markers in Table I from the centromere (top) is according to the combined data for RFLP mapping using reference families $(4,11)$, physical mapping data from hybrid cell panels, and long-range restriction enzyme mapping with pulsed-field gel electrophoresis $(13,14)$. Seven "anchor" markers indicated in bold are those probes mapped by linkage in reference families (11) (Fig. 1). In the following, the same gene order is used in tables and figures.

Only marker systems that gave significant linkage to MEN1 are listed in Table I. As shown, three marker systems (D1 1S146, D1 1S751, and INT2) with two separate 2-allele RFLP's were combined to 4-allele systems by haplotyping. For the markers D11S288 and D11S149 previous linkage data in reference families have shown a very close linkage with no meiotic crossover between these two loci $(4,11)$. Similarly, we have not detected any recombinations between these markers in our MEN1 families. Therefore, these two markers were also combined by haplotyping, in order to increase their relative information content. Haplotyping of the two markers for the PYGM locus and the combined D1 1S97 + D1 1S146 markers is also possible, although we have chosen to analyze them separately since the information content was high for each one of these markers.

Methods and conditions for DNA preparation, Southern blotting, hybridization to radioactively labeled DNA probes, and autoradiography were as described (14). Several of the markers used contained repetitive DNA elements (marked with ${ }^{\S}$ in Table I), and were therefore hybridized in the presence of denatured, sonicated human DNA.

Family and genotype data were typed into the linkage data managing computer database "LINKSYS." Two-point linkage between MEN1 and different marker loci was calculated with the "LIPED" computer program, assuming full penetrance for MEN1 under the strict diagnostic criteria outlined above.

\section{Results}

Linkage to MEN1. A number of DNA markers mapped within, or close to, chromosome bands 11 q11-q13 were tested for linkage to the disease in six MEN1 families. 13 marker systems gave significant lod scores (Table II). Three pedigrees from Sweden (LU, KA, and EZ) previously outlined in the original mapping of MEN1 (3) were reanalyzed, since some of the marker systems used may be more informative if the experimental conditions are adjusted. In addition, two new Swedish families (AX and RA) (Fig. 2) and part of one large MEN1 pedigree from Tasmania were studied (15). The total number of individuals genotyped was 124, including 34 MEN1 cases from Tasmania and 25 cases from the Swedish pedigrees.

The two-point linkage data of MEN1 to the different genetic markers are summarized in Table II. 13 different marker systems gave lod scores above the conventional cut-off level of 3.1. The marker systems break down into three groups based



Figure 1. Map of the MEN1 region on chromosome 11 determined by different mapping strategies. Seven anchor markers are indicated to the left, and the genetic linkage distances in percentage meiotic recombinations between these markers, according to reference 11 , are given. The new markers are placed in the gene order and in relation to the anchor markers, as determined by physical mapping (references 13 and 14). The linkage distances in percentage crossing over to MEN1 are from Table II.

on meiotic crossovers. Four marker systems are centromeric to the MEN1 locus, D11S288/D11S149, CD20, D11S480, and PGA. In our previous linkage studies, we did not find any crossovers between MEN1 and PGA, but this was now found in the Tasmanian family using the new cosmid probe for PGA (cCLPGA12).

Four marker systems are telomeric to MEN1-D11S97, D1 1S146, D1 1S751, and INT2. Between these groups of flanking marker systems there are five probes closely linked to MEN1, for which we found no crossing over. The probe pMCMP1 for the PYGM locus shows the highest lod score (10.80) at a peak recombination distance of $\theta=0$, in agreement with our previous studies $(3,4)$. Four newly isolated cosmid probes, cCL15 for PYGM, D1 1S427, D1 1S449, and D1 1S750, gave lod scores in the range of 3-5. The lower lodscores for these markers are in part due to the fact that they are less informative than the multiallele VNTR detected by the PYGM probe pMCMP1.

DNA testing for MEN1. To illustrate how DNA testing can be used in clinical situations, we selected six Swedish nuclear families that had undergone extensive biochemical testing for MEN1. These families are outlined in Fig. 2. In each of these families, one of the parents and at least one child are affected with MEN1. Based on the genotypes determined for different marker systems linked to MEN1 in two affected members in each kindred, we can outline the most likely haplotype carrying the MEN1 allele in each affected parent. For the KA-2 and KA-3 kindred, the first child is unaffected according to our 
Table II. Lod Scores for Linkage of MEN1 to Different Loci within Chromosome Region 11q11-q13

\begin{tabular}{|c|c|c|c|c|c|c|c|c|c|c|c|}
\hline \multirow[b]{2}{*}{ Marker system } & \multirow[b]{2}{*}{0} & \multirow[b]{2}{*}{0.001} & \multirow[b]{2}{*}{0.01} & \multicolumn{3}{|c|}{ Recombination fraction $(\theta)$} & \multirow[b]{2}{*}{0.20} & \multirow[b]{2}{*}{0.30} & \multirow[b]{2}{*}{0.40} & \multirow[b]{2}{*}{$\theta$} & \multirow[b]{2}{*}{$Z$} \\
\hline & & & & 0.05 & 0.10 & 0.15 & & & & & \\
\hline (D11S288/D11S149)* & $-\infty$ & 4.04 & 5.88 & 6.56 & 6.21 & 5.57 & 4.78 & 2.99 & 1.17 & 0.05 & 6.56 \\
\hline CD20 & $-\infty$ & 2.39 & 3.27 & 3.43 & 3.06 & 2.57 & 2.04 & 1.00 & 0.23 & 0.03 & 3.48 \\
\hline D11S480 & $-\infty$ & 0.58 & 2.59 & 3.82 & 3.96 & 3.71 & 3.27 & 2.04 & 0.66 & 0.09 & 3.98 \\
\hline PGA (cCLPGA12) & $-\infty$ & 2.03 & 2.93 & 3.22 & 3.01 & 2.66 & 2.26 & 1.37 & 0.50 & 0.04 & 3.23 \\
\hline PYGM (pMCMP1) & 10.80 & 10.78 & 10.63 & 9.87 & 8.82 & 7.69 & 6.50 & 3.99 & 1.52 & 0 & 10.80 \\
\hline PYGM (cCL15) & 4.26 & 4.25 & 4.17 & 3.85 & 3.45 & 3.04 & 2.62 & 1.70 & 0.72 & 0 & 4.26 \\
\hline D11S427 & 4.78 & 4.77 & 4.68 & 4.28 & 3.78 & 3.26 & 2.75 & 1.72 & 0.70 & 0 & 4.78 \\
\hline D11S750 & 3.57 & 3.56 & 3.52 & 3.27 & 2.91 & 2.49 & 2.03 & 1.08 & 0.25 & 0 & 3.57 \\
\hline D11S449 & 3.39 & 3.38 & 3.28 & 2.84 & 2.33 & 1.86 & 1.43 & 0.71 & 0.19 & 0 & 3.39 \\
\hline D11S97 & $-\infty$ & 4.99 & 7.77 & 8.86 & 8.46 & 7.64 & 6.64 & 4.34 & 1.90 & 0.05 & 8.86 \\
\hline D11S146 & $-\infty$ & 7.63 & 9.43 & 9.92 & 9.32 & 8.40 & 7.33 & 4.90 & 2.25 & 0.04 & 9.96 \\
\hline D11S751 (cCL8/clone8) & $-\infty$ & 4.94 & 5.79 & 5.79 & 5.21 & 4.49 & 3.72 & 2.15 & 0.75 & 0.03 & 5.92 \\
\hline INT2 & $-\infty$ & 1.19 & 4.99 & 6.84 & 6.82 & 6.26 & 5.45 & 3.48 & 1.40 & 0.07 & 6.94 \\
\hline
\end{tabular}

* Combined haplotype of the two markers.

criteria. Hence, in these kindred the DNA-based testing uses the first affected child as reference case.

For each additional child, the carrier status for MEN1 can now be inferred from its haplotype of these marker systems. This is illustrated in the RA kindred, where the first daughter who inherited MEN1 from her mother also inherited the 2-allele for the PYGM marker cCL15, and the 3-allele for D1 1S427. In addition, the 1-allele for PGA and the 3-allele for D11S97 were linked to the disease. The younger brother had inherited the same haplotype as his affected sister. The most likely interpretation of these data is that he has inherited a nonrecombinant stretch of this chromosome region, including all loci from PGA to D11S97. Hence, this would also include the MEN1 mutation. The predicted MEN1 diagnosis is confirmed for this boy, who underwent surgery for primary hyperparathyroidism, and treated for prolactinoma. Since four markers were used, two very close to MEN1 and two flanking the disease locus, this family illustrates not only the use of the very closely linked markers cCL15 and D11S427 for genetic testing, but also that meiotic crossovers close to MEN1 could be excluded.

If a crossover had occurred in the second child, this would only escape detection if the first child also had inherited a recombinant chromosome with the crossover point between the same two markers. In this case then, an incorrect prediction of the disease predisposition in the second child could only occur if both children had inherited recombinant chromosomes. The probability for this is $<0.003$, based on the linkage map shown in Fig. 1. Furthermore, this would only result in incorrect prediction in the instance that the one of these crossover events had occurred between the disease locus and the two marker loci PYGM and D1 1S427, for which no recombinant has yet been found. In the RA family, the third and still unaffected 24-yr-old child (marked with ?) is predicted to carry the disease gene based on the genotype data (Fig. 2).

In each of the following kindred outlined in Fig. 1, we show
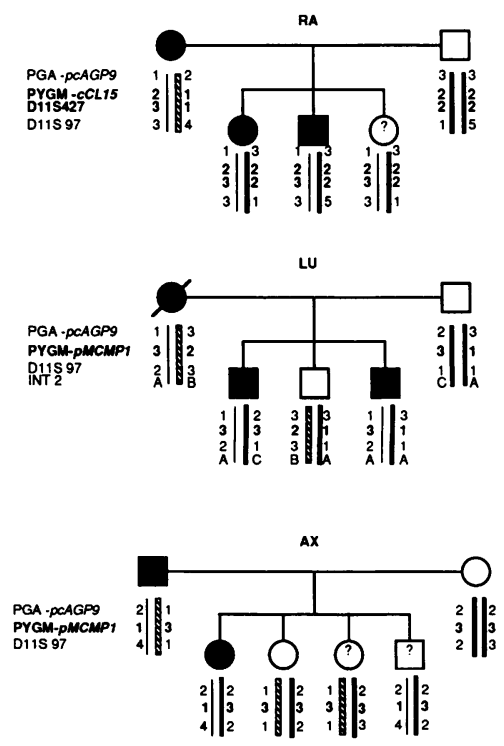

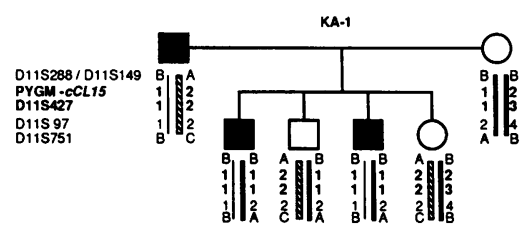

KA-2

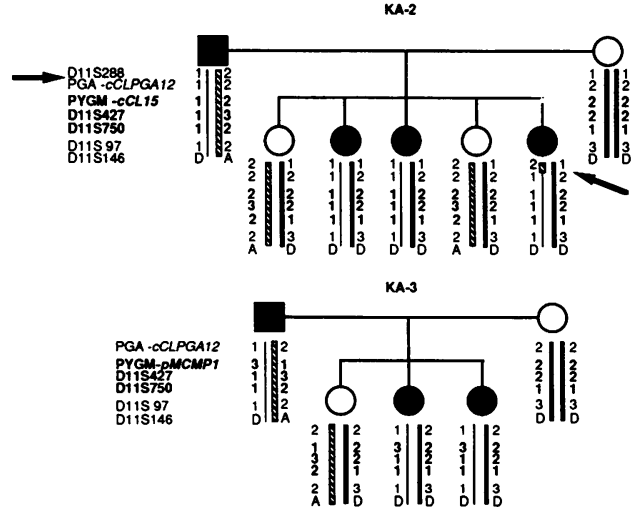

Figure 2. Pedigrees illustrating DNA based predictive testing for MEN1. Filled symbols indicate affected family members. Numbers below each individual indicate the genotypes for the informative marker systems, listed next to the symbol of the carrier parent. The haplotypes for each individual are depicted along an illustrated chromosome segment, with the inferred mutant chromosome indicated by a thin line, and the wild type chromosome of the carrier parent hatched. The arrows at kindred KA-2 indicate the point of meiotic crossover in the fifth daughter. KA-1, KA-2, and $\mathrm{KA}-3$ are subsets of the KA pedigree (3). Blood samples from the mother in the LU family was obtained before her death in 1989. 
that the DNA testing for MEN1 is based on at least one marker which shows no crossover with the disease locus, and at least one marker flanking the disease on each side of the disease locus. Thus, we excluded crossovers close to MEN1 in each of these kindreds. As illustrated in the KA-2 kindred, a meiotic crossover had occurred in the fifth daughter between D1 1S288 and PGA. Since the point of crossover could be located in this girl, we could then use six alternative markers beyond this point, PGA, PYGM, D11S427, D11S750, D11S97, and D1 1S146, and found no evidence for crossing over for any of these markers.

The set of six families outlined in Fig. 2 illustrate how DNA testing for MEN1 would be possible to use for predictive testing. In 16 offsprings (all children except one in each family) we know the outcome of 13 based on clinical and biochemical data. In all these cases the DNA test was totally conclusive based on a set of at least one closely linked probe, $(\theta=0 \%)$ and two flanking probes in all instances (Fig. 2). Hence, the set of markers described here (Table I and II) provide a useful and accurate means for predictive testing of MEN1. Since the DNA test can be applied at any age, providing the family structure permits linkage analysis, it can predate the biochemical and clinical presentation of the disease by two to three decades with very high accuracy $(>99.5 \%)$. Such predictions can be made for the third child (age 24) in the RA kindred who is deduced to carry the MEN1 predisposition. Similarly the youngest daughter (age 26) in the AX kindred is predicted unaffected, while her younger brother (age 25), still unaffected, is a gene carrier.

\section{Discussion}

In this report, we have presented an extensive family study using five RFLP markers closely linked to MEN1, with no meiotic recombinations with the disease locus. These five closely linked probes are then flanked by four marker systems on each side, all located within $7 \mathrm{cM}$ from the disease gene, based on combining genetic linkage in reference families and our own linkage data in MEN1 families (Table II). The 17 probes studied in this report include 7 anchor markers which have been ordered by multipoint linkage analysis (11). All probes used here have also been mapped physically within the 11q12-q13 region (12-14). The gene orders, as determined by physical mapping $(13,14)$ and genetic linkage data $(4,11)$ are in full agreement with the data presented here.

As illustrated in Fig. 2, the DNA probes linked to MEN1 provide a set of linkage markers that will enable accurate and reliable premorbid diagnosis of the disease predisposition already at birth, providing the family structure permits linkage analysis. There are three main causes of incorrect results from DNA-based testing of inherited diseases: $(a)$ meiotic crossing over between the marker and the disease loci, $(b)$ incorrect diagnosis of an individual; and (c) mixing of samples. The latter two can only be managed by correct clinical and laboratory procedures, while meiotic crossovers are a serious problem when the markers are not closely linked to the disease, or the number of useful markers is restricted. The set of 17 DNA probes linked to MEN1 provides a useful and reliable tool to eliminate crossing over as cause of incorrect prediction of MEN1. This includes five closely linked probes that are flanked by a similar set of markers on each side of the disease locus. Hence, we are not only able to determine the linkage phase, using at least one very close marker system (with no crossover with MEN1), but also to detect if a meiotic crossover event has occurred within the chromosomal region flanking the MEN1 locus. Until the disease gene has been cloned, predictive DNA testing for MEN1 should be supported by at least one closely linked marker and one flanking marker on each side of the disease locus, in order to avoid incorrect diagnosis due to meiotic crossing over. In this instance, the reliability of the prediction will exceed $99.5 \%$, which is a common level for inaccuracies due to sample mixing or incorrect diagnosis of patients.

In conclusion, the marker systems in Table II that show recombination fractions of- $\infty$ are located outside the MEN1 region based on meiotic crossovers. Therefore, we would like to stress that predictive testing for MEN1 should not be based on linkage to these markers alone. Instead, these markers are to be used as indicators of crossing over close to MEN1, in order to identify matings where predictive testing can not be considered reliable.

Finally, one should not exclude the possibility that meiotic crossing over may occur between MEN1 and any of the five markers closest to the disease locus. In a clinical test situation, this will not pass undetected if the setup illustrated in Fig. 2 is used. On the other hand, any such crossover will be a key finding for further locating the MEN1 gene within this region, and will facilitate the final cloning of the MEN1 gene.

\section{Acknowledgments}

We would like to acknowledge Marilyn Walters for establishing cell lines from the Tasmanian pedigree.

The work was supported by grants from Axel and Margaret Ax:son Johnson's Fond, the Swedish Medical Research Council, the Swedish Cancer Society, Paul Mackay Bolton Foundation, Tasmanian Cancer Committee, the Australian National Health and Medical Research Council, Torsten and Ragnar Söderberg's, and Lars Hierta's and Magnus Bergwall's Fond.

\section{References}

1. Wermer, P. 1963. Endocrine adenomatosis and peptic ulcer in a large kindred. Am. J. Med. 35:205.

2. Brandi, M. L., S. J. Marx, G. D. Aurbach, and L. A. Fitzpatrick. 1987. Familial multiple endocrine neoplasia type 1; A new look at pathophysiology. Endocr. Rev. 8:391-405.

3. Larsson, C., B. Skogseid, K. Öberg, Y. Nakamura, and M. Nordenskjöld. 1988. Multiple endocrine neoplasia type 1 gene maps to chromosome 11 and is lost in insulinoma. Nature (Lond.) 332:85-87.

4. Nakamura, Y., C. Larsson, C. Julier, C. Byström, B. Skogseid, S. Wells, K. Öberg, M. Carlson, T. Taggert, P. O'Connel, P. Leppert, J. M. Lalouel, M. Nordenskjöld, and $R$. White. 1989. Localization of the genetic defect in multiple endocrine neoplasia type 1 within a small region of chromosome 11. Am. J. Hum. Genet. 44:751-755.

5. Friedman, E., K. Sakaguchi, A. E. Bale, A. Falchetti, E. Streeten, M. B. Zimering, L. S. Weinstein, W. O. McBride, Y. Nakamura, M. L. Brandi, J. A. Norton, G. D. Aurbach, A. M. Spiegel, and S. J. Marx. 1989. N. Engl. J. Med. 321:213-218.

6. Thakker, R. V., P. Bouloux, C. Wooding, K. Chotai, P. M. Broad, N. K. Spurr, G. M. Besser, and J. L. H. O'Riordan. 1989. Association of parathyroid tumors in multiple endocrine neoplasia type 1 with loss of alleles on chromosome 11. N. Engl. J. Med. 321:218-224.

7. Byström, C., C. Larsson, C. Blomberg, K. Sandelin, U. Falkmer, B. Skogseid, K. Öberg, S. Werner, and M. Nordenskjöld. 1990. Localization of the gene for multiple endocrine neoplasia type 1 to a small region within chromosome 11 q13 by deletion mapping in tumors. Proc. Natl. Acad. Sci. USA. 87:1968-1972.

8. Teh, B. T., N. K. Hayward, S. Wilkinson, G. M. Woods, D. Cameron, and 
J. J. Shepherd. 1990. Clonal loss of Int-2 alleles in sporadic and familial pancreatic endocrine tumours. Br. J. Cancer. 62:253-254.

9. Knudson, A. G. 1978. Retinoblastoma: a prototypic hereditary neoplasm. Semin. Oncol. 5:57-60.

10. Skogseid, B., B. Eriksson, G. Lundqvist, L. E. Lörelius, J. Rastad, L. Wide, E. Wilander, G. Åkerström, and K. Öberg. 1991. Multiple endocrine neoplasia type 1-A ten year prospective screening study in four kindreds. J. Clin. Endocrinol. Metab. 73:281-287.

11. Julier, C., Y. Nakamura, M. Lathrop, P. O'Connell, M. Leppert, M. Litt, T. Mohandas, J. M. Lalouel, and R. White. 1990. Detailed map of the long arm of chromosome 11. Genomics. 7:335-345.

12. Tokino, T., E. Takahashi, M. Mor, A. Tanigami, T. Glaser, J. W. Park, C. Jones, T. Hori, and Y. Nakamura. 1991. Isolation and mapping of 62 new RFLP markers on human chromosome 11. Am. J. Hum. Genet. 48:258-268.

13. Janson, M., C. Larsson, B. Werelius, C. Jones, T. Glaser, Y. Nakamura, P. Jones, and M. Nordenskjöld. 1991. Detailed physical map of human chromosomal region 1 1q12-13 shows high meiotic recombination rate around the MEN I locus. Proc. Natl. Acad. Sci. USA. 88:10609-10613.
14. Larsson, C., G. Weber, E. Kvanta, K. Lewis, M. Janson, C. Jones, T. Glaser, G. Evans, and M. Nordenskjöld. 1991. Isolation and mapping of polymorphic cosmid clones used for sublocalization of the MEN1 locus. Hum. Genet. In press.

15. Shepherd, J. J. 1985. Latent familial multiple endocrine neoplasia in Tasmania. Med. J. Aust. 142:385-397.

16. Shepherd, J. J. 1991. The natural history of MEN1 which may be highly unrecognised rather than highly uncommon. Arch. Surg. In press.

17. Kidd, K. K., A. M. Bowcock, J. Schmidtke, R. K. Track, F. Ricciuti, G. Hutchings, A. Bale, P. Pearson, and H. Willard. 1989. Human gene mapping 10. Cytogenet. Cell Genet. 51:622-947.

18. Williamson, R., A. Bowcock, K. Kidd, P. Pearson, J. Schmidtke, H. S. Chan, M. Chipperfield, D. N. Cooper, J. Hewitt, F. Lewitter, B. Maidak, M. Quitt, F. Ricciuti, and R. K. Track. 1990. Human gene mapping 10.5. Cytogenet. Cell Genet. 55:457-778.

19. Rabbitts, P. H., J. Douglas, P. Fisher, E. Nacheva, A. Karpas, D. Catovsky, J. V. Melo, R. Baer, M. A. Stinson, and T. H. Rabbitts. 1988. Chromosome abnormalities at 11q13 in B cell tumors. Oncogene. 3:99-103. 